Andere Oberverwaltungsgerichte fordern allerdings weiterhin die Rückkehr der Ehepartner ins Herkunftsland und den Sprachnachweis und Visumserwerb zum Zweck des Ehegattennachzugs bei den dortigen Auslandsvertretungen. ${ }^{47}$

\section{Differenzierung der Sprachanforderungen nach Aufenthalts- status und Staatsangehörigkeit}

Verfassungsrechtlich problematisch ist auch die Differenzierung der Sprachanforderungen nach dem Aufenthaltszweck, die dazu führt, dass der zum Zweck der Beschäftigung einreisende Ausländer vor Einreise keine Sprachkenntnisse nachweisen muss, die nachziehende Ehepartnerin aber schon. ${ }^{48}$ Ähnlich problematisch ist die Differenzierung zwischen dem Nachzug zu ausländischen oder deutschen Wissenschaftlern oder zu ausländischen oder deutschen Selbständigen. ${ }^{49}$ Die Nähe zum Grundrecht von Ehe und Familie, eventuell auch zum geschlechtsspezifischen Diskriminierungsverbot, stellt strenge Anforderungen an die gebotene Gleichbehandlung, denen die Regelungen des $\mathbb{} 30$ Abs. 1 AufenthG nicht gerecht werden. Auch das Argument, gerade der Nachzug zu deutschen Ehegatten erfordere wegen des privilegierten Aufenthaltsstatus Deutschkenntnisse, vermag die genannten Differenzierungen nicht zu rechtfertigen. Wesentlich konsistenter wäre ein Konzept, das nachziehende Ehepartner ausnahmslos auf den Nachweis und gegebenenfalls Erwerb ausreichender Sprachkenntnisse nach der Einreise verpflichtet.

\section{Resümee}

Die Integrationsbedingung ausreichender Deutschkenntnisse bleibt damit problematisch. Die Integrationsbedingung ist unverhältnismäßig, insbesondere dann, wenn die Infrastruktur oder die persönlichen Verhältnisse im Herkunftsland den Spracherwerb erschweren. Sie ist außerdem unklar, was den Standard und Nachweis der sprachlichen Fähigkeiten, aber auch, was die Möglichkeiten des Erwerbs im Bundesgebiet betrifft. Der deutsche Gesetzgeber sollte auf diese Integrationsbedingung im Ehegattennachzug deshalb verzichten. Der mit dem Instrument verfolgte - ohne Zweifel wünschenswerte und förderungswürdige - Zweck des Spracherwerbs lässt sich durch das Angebot von Integrationskursen, ggf. auch durch die Verpflichtung zur Teilnahme erreichen. Der Einwand, der Spracherwerb sei nach Zuzug ins Bundesgebiet nur noch schwer durchzusetzen, ist wenig stichhaltig. Das Beispiel des Aufnahme- und Integrationsvertrags in Frankreich zeigt, dass Instrumente zur Verfügung stehen, mit denen sich die gesetzlichen Ziele auf andere, für die Ehepaare mildere Weise durchsetzen lassen.

47 Vgl. HessVGH, B. v. 22.9.2008 - 1 B 1628/o8, juris; OVG RheinlandPfalz, B. v. 20.4.2009-7 B 10037/og, juris; OVG Lüneburg, B. v. 27.7.2009-11 ME 171/og, juris; anders noch OVG Lüneburg, B.v. 28.8.2008-11 ME 131/08, juris.

48 Kingreen, ZAR 2007, 13 (19).

49 Ausführlich Henning J. Thomas, SächsVBI. 2009, 56 (59 f.).

\title{
Probleme der Vereinbarkeit von Scharia-geprägtem Familienrecht mit deutschem Recht
}

Beatrix Weber-Monecke,

Richterin am Bundesgerichtshof, Karlsruhe

Zunächst möchte ich einen kurzen Streifzug durch islamisches Familienrecht, insbesondere durch das Scheidungsrecht, unternehmen. Im Islam ist die Scheidung (arab.: talaq) das Auflösen eines Rechtsverhältnisses, das durch den privatrechtlichen Vertrag der Eheschließung zustande gekommen ist. ${ }^{1}$ Die Scheidung ist eine einseitige Rechtshandlung, die vom Ehemann oder einem Stellvertreter vorgenommen werden kann und die zu ihrer Gültigkeit in Anwesenheit von zwei Zeugen erfolgen muss. Eine Ehefrau kann die Scheidung nur begehren, wenn ihr Ehemann ihre zwingenden Rechte verletzt hat. ${ }^{2}$

\section{Überblick über Scharia-geprägtes Scheidungsrecht am Bei- spiel des islamisch-iranischen Rechts \\ Das iranische Recht, mit dem ich mich exemplarisch befassen will, sieht ausschließlich die gerichtliche Ehescheidung vor. Es gibt sogenannte zivile Sondergerichte, die für Personenstands- sachen, daher insbesondere auch für Scheidungen, zuständig}

sind. Dem Gericht sitzen männliche Richter vor; daneben können weibliche Beraterinnen berufen werden, was in der Praxis dem Vernehmen nach auch geschieht. Dem Antrag auf Scheidung folgt ein Verfahren, in dem Schiedsrichter ernannt werden, die die Parteien zu beraten und auf die Beilegung der Streitigkeiten hinzuwirken haben. Dieses Verfahren ist unabhängig davon einzuhalten, ob ein Mann oder eine Frau die Scheidung begehrt. ${ }^{3}$ Im iranischen ZGB ist die Scheidung für Muslime in Anlehnung an das islamisch-schiitische Recht als Mannesrecht geregelt. Ein Mann kann ohne Vorliegen und Angabe von Gründen seine Frau verstoßen. ${ }^{4}$ Das Scheidungsbegehren einer Frau ist dagegen davon abhängig, dass Scheidungsgründe vorliegen. Deshalb spielt das materielle Scheidungsrecht insbesondere in dieser Hinsicht eine Rolle.

1 Zum Eheschließungsvertrag im iranischen Recht: Bergmann/Ferid/ Henrich/Enayat, Internationales Ehe- und Kindschaftsrecht, Länderteil Iran, Ergänzungslieferung 158, $37 \mathrm{f}$.

2 Yassari, FamRZ 2002, 1088; Elwan/Menhofer STAZ 2005, 168, 172 f.

3 Vgl. Yassari, FamRZ 2002, 1088, 1089 f.; Bergmann/Ferid/Henrich/ Enayat, a.a.O., $63 \mathrm{ff}$.

4 Art. 1133 iran. ZGB. 


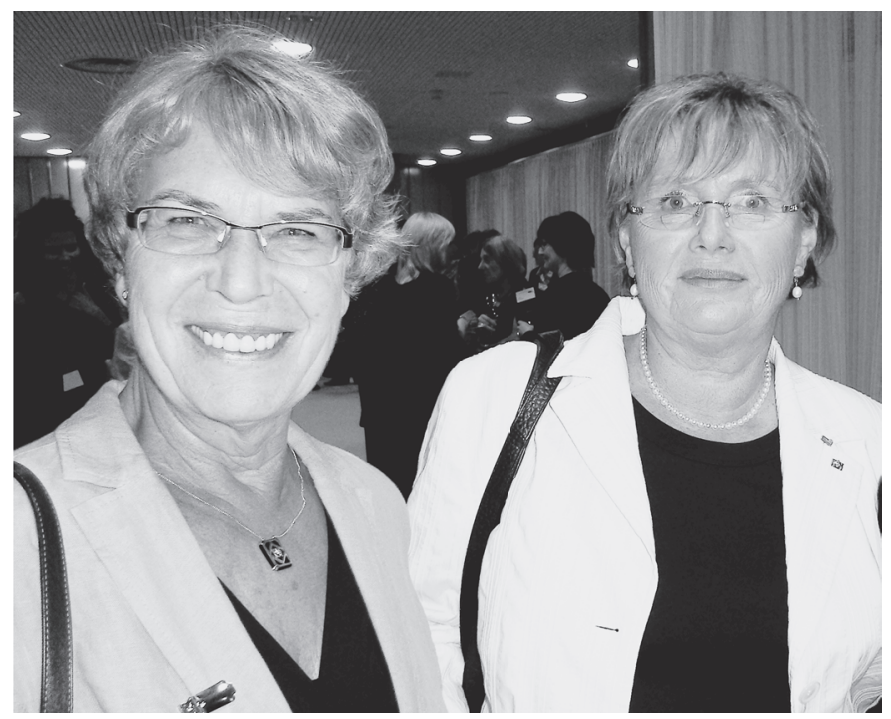

$\Delta$ Vors. Richterin am LAG i.R. Ingrid Weber (links) mit Richterin am EGMR Dr. h.c. Renate Jaeger (Foto: AG).

Das iranische ZGB enthält folgende Scheidungsgründe, auf die eine Frau sich stützen kann:

- Verschollenheit des Ehemannes ${ }^{5}$

Dieser Scheidungsgrund bedarf im Rahmen des vorliegenden Überblicks keiner Erläuterung; es sei nur angemerkt, dass bestimmte Fristen einzuhalten sind.

- Weigerung des Ehemannes, Unterhalt zu leisten ${ }^{6}$

Nach iranischem ZGB ist der Mann der Haushaltsvorstand; ${ }^{7}$ Unterhalt wird nur von ihm geschuldet, und zwar unabhängig von der Unterhaltsbedürftigkeit der Ehefrau. ${ }^{8}$ Bei Leistungsverweigerung kann die Ehefrau das Gericht anrufen, um den Ehemann zur Zahlung zu zwingen. Ist er leistungsfähig, kann er zur Zahlung verurteilt werden. Befolgt er die Aufforderung zur Zahlung nicht, kann die Zwangsvollstreckung veranlasst und die Ehefrau aus dem Erlös befriedigt werden. ${ }^{9}$ Im Fall der Befriedigung hat die Ehefrau kein Scheidungsrecht, ein solches steht ihr nur bei fruchtloser Vollstreckung zu. ${ }^{10}$ Ist der Ehemann dagegen nicht zur Zahlung in der Lage, hat die Ehefrau sofort das Recht, die Scheidung zu beantragen. ${ }^{11}$

- Bedrängnis der Ehefrau (osr wa haraj) $)^{12}$

„Osr wa haraj“ ist dem Koran entnommen. Die Auslegung dieses Begriffes ist auch im Iran umstritten. Er hat etwa die Bedeutung von „Härte“ und „Widrigkeit", „,in Not“ oder „in eine kritische Lage“ geraten. ${ }^{13}$ Im rechtlichen Zusammenhang ist darunter eine sehr starke Erschwernis, eine große Mühsal, ein Leben unter Umständen, die dem Wesen einer Ehe nicht entsprechen, zu verstehen. Die Ehefrau gerät in große Bedrängnis und sieht sich in dieser Situation dem Vorwurf ausgesetzt, ihren Pflichten nicht nachgekommen zu sein. ${ }^{14}$

Die Beurteilung, ob „osr wa haraj“ vorliegt, obliegt der Beweiswürdigung des Gerichts. ${ }^{15}$ Die schlichte Behauptung einer solchen Situation reicht nicht aus. Erforderlich sind etwa polizeiliche Unterlagen über Misshandlungen oder
Zeugen hierfür. Auch die Umstände, die zu der Situation geführt haben, müssen bewiesen werden. ${ }^{16}$

$\mathrm{Zu}$ ergänzen ist noch, dass ungeachtet des Vorliegens von „osr wa haraj“ der Grundsatz bestehen bleibt, dass die Scheidung das Recht des Ehemannes ist. Erst wenn die Aufrechterhaltung der Ehe für die Frau eine große Härte bedeutet und mit Schwierigkeiten verbunden ist und der Mann die Frau nicht verstößt, kann sie die Scheidung beantragen. Der Richter verpflichtet den Mann zur Verstoßung seiner Frau. Sollte sich der Mann trotzdem weigern, spricht der Richter die Scheidung der Ehe aus. ${ }^{17}$

- Vertraglich vereinbarte Scheidungsgründe

Nach iranischem Recht kann es darüber hinaus vertraglich vereinbarte Scheidungsgründe geben. Da die Eheschließung - wie schon ausgeführt - ein zivilrechtlicher Vertrag ist, für den in den gesetzlichen Grenzen das Prinzip der Vertragsfreiheit gilt, können die künftigen Ehegatten ihre gegenseitigen Rechte und Pflichten im Eheschließungsvertrag näher regeln. Dabei kann auch der Ehefrau das Recht eingeräumt werden, unter bestimmten Umständen die Scheidung zu beantragen. Dieses Recht ist jedoch wiederum an Gründe gebunden, die weder dem Wesen der Ehe widersprechen noch unmoralisch oder ungesetzlich sein dürfen. Kann die Ehefrau dem Gericht das Vorliegen dieser Gründe beweisen, so hat sie die Vollmacht - mit dem Recht zur Bestellung eines Vertreters -, die Ehe unwiderruflich aufzulösen. ${ }^{18}$

Schon aus dieser kurzen Darstellung ergibt sich, dass das iranische Scheidungsrecht anders strukturiert ist als das deutsche, das für beide Ehegatten gleichermaßen die Möglichkeit vorsieht, die Scheidung ihrer Ehe durch richterliches Gestaltungsurteil zu erreichen, wenn sie gescheitert ist.

\section{Vermögensrechtliche Scheidungsfolgen}

Auch im Bereich der vermögensrechtlichen Scheidungsfolgen bestehen gravierende Unterschiede. In Betracht kommen allein nachehelicher Unterhalt, die Herausgabe der Morgengabe, die Rückforderung der Mitgift und eine Abfindung für während der Ehe erbrachte Dienstleistungen. ${ }^{19}$

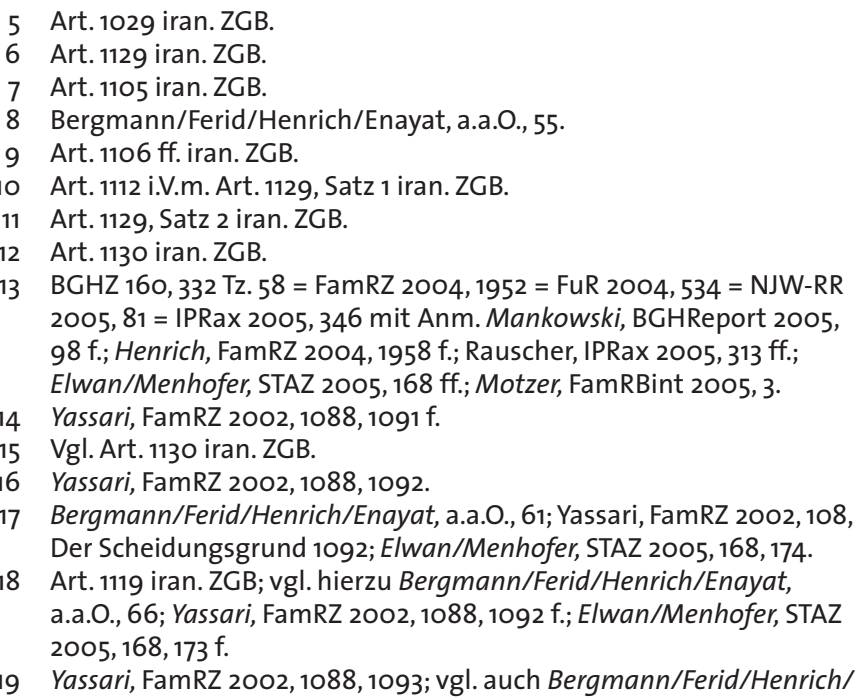

19 Yassari, FamRZ 2002, 1088, 1093; vgl. auch Bergmann/Ferid/Henrich/ Enayat, a.a.O., 51. 


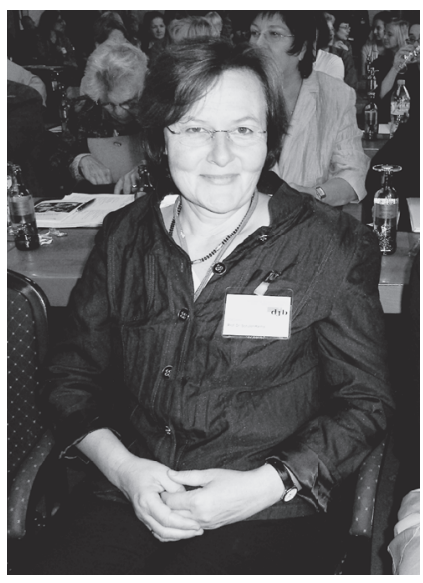

$\triangle$ Prof. Dr. Margarete SchulerHarms (seit 2009 Vors. der djb Kommission Recht der sozialen Sicherung Familienlastenausgleich) (Foto: AG)

- Unterhalt

Der Unterhaltsanspruch erscheint aus unserer Sicht stark eingeschränkt. Er besteht für die Zeit nach der Scheidung nur während der sogenannten Wartezeit, die mit der Eintragung der Scheidung zu laufen beginnt, ${ }^{20}$ und umfasst Ernährung, Bekleidung, Hausrat usw. ${ }^{21}$ Eine gesetzliche Unterhaltspflicht über die Wartezeit hinaus kennt das islamisch-iranische Recht dagegen nicht.

- Morgengabe

Die Vereinbarung einer Morgengabe (mahr) ist bei der Eheschließung üblich. Es handelt sich um einen Vermögenswert, der der Frau zur alleinigen Verfügung steht und ihr in diesem Rahmen wirtschaftliche Unabhängigkeit geben soll. Wird die Morgengabe bei der Eheschließung oder während der Ehe nicht ausgehändigt, ist sie spätestens bei der Scheidung zu zahlen, unabhängig davon, wer den Scheidungsantrag stellt. ${ }^{22}$

\section{- Abfindung}

Die Abfindung für erbrachte - nach religiösen Vorschriften nicht zu ihren Pflichten zählende - Dienstleistungen setzt dagegen voraus, dass der Ehemann die Scheidung betreibt, der Ehefrau keine Pflichtverletzungen anzulasten sind und sie an der Ehe festhalten will. ${ }^{23} 24$ Der zu entrichtende Betrag entspricht nicht dem, was nach deutschem Recht als Zugewinnausgleich zu zahlen wäre. Die jeweiligen Vermögen werden nicht einander gegenübergestellt und die Differenz ausgeglichen. Vielmehr ist eine Art Gehaltsabfindung zu entrichten, deren Höhe durch einen gerichtlichen Sachverständigen ermittelt wird. $\mathrm{Zu}$ berücksichtigen sind etwa das Einkommen des Ehemannes und der Lebensstandard in der Familie der Ehefrau. ${ }^{25}$

- Rückforderung der Mitgift

Vermögenswerte, die die Ehefrau als Mitgift erhält, bleiben - ebenso wie das Vermögen, das sie vor der Ehe erworben hat - auch nach der Eheschließung ihr Eigentum. Nach der Scheidung kann die Ehefrau ihr Eigentum, also auch die Mitgift, herausverlangen. ${ }^{26}$

Angesichts dieser stark unterschiedlichen materiell- und verfahrensrechtlichen Regelungen liegt es auf der Hand, dass die Vereinbarkeit von Scharia-geprägtem Familienrecht und deutschem Recht Probleme bereiten kann.

\section{Probleme der Vereinbarkeit am Beispiel des BGH-Urteils vom 6.10.2004 - XII ZR 225/o1 - BGHZ 160, 332 ff.}

Der XII. Zivilsenat des Bundesgerichtshofs hatte sich mit solchen Problemen in dem folgenden Fall ${ }^{27}$ zu befassen: Die Ehegatten, iranische Staatsangehörige schiitischer Religion, hatten 1987 in Teheran geheiratet und bei der Eheschließung zugleich weitere vertragliche Vereinbarungen getroffen. Sie leben seit 1995 in Deutschland. Aus der Ehe sind zwei Kinder hervorgegangen, die damals beide noch minderjährig waren. Die Ehefrau hatte unter anderem die Scheidung der Ehe begehrt. Sie hatte geltend gemacht, ihr Ehemann habe nie genügend Zeit für die Familie aufgebracht. Inzwischen habe er sich auch einer anderen Frau zugewandt. Außerdem hatte sie sich auf die Verweigerung des Unterhalts gestützt.

Das Amtsgericht hatte den Scheidungsantrag in den Antrag umgedeutet, den Ehemann zu verpflichten, die Scheidungsformel (Verstoßung) auszusprechen, diesem Antrag stattgegeben und festgestellt, dass die Ehe damit aufgelöst sei. Auf die Berufung des Ehemannes wurde der Scheidungsantrag abgewiesen. Dagegen hatte die Ehefrau Revision eingelegt.

\section{Internationale Zuständigkeit}

Zunächst stellt sich in Verfahren mit Auslandsbezug die Frage nach der internationalen Zuständigkeit der deutschen Gerichte. Diese ergibt sich seit dem Inkrafttreten der EG-EheVO primär aus dieser Verordnung. Nur für Verfahren, die - wie hier - vor dem Inkrafttreten der Verordnung am 1. März 2001 eingeleitet worden sind oder aus anderen Gründen nicht in den Anwendungsbereich der Verordnung fallen, richtet sich die internationale Zuständigkeit nach deutschem Zivilprozessrecht, und zwar nach $\$$ 606a ZPO. Danach sind die deutschen Gerichte z.B. international zuständig, wenn beide Ehegatten ihren gewöhnlichen Aufenthalt im Inland haben. Diese Voraussetzung war hier gegeben. In einem solchen Fall - d.h. bei gewöhnlichem Aufenthalt beider Parteien im Inland - verlangt das Gesetz auch keine Prognose darüber, ob die deutsche Entscheidung nach dem Recht des gemeinsamen Heimatstaates der Parteien anerkannt würde. ${ }^{28}$ Dasselbe gilt übrigens auch nach der EG-EheVO. ${ }^{29}$ Aber selbst wenn die fehlende Anerkennungsfähigkeit offensichtlich wäre, würde das der internationalen Zuständigkeit nicht entgegenstehen. Letzteres ist hier

20 Einzelheiten bei Bergmann/Ferid/Henrich/Enayat, a.a.O., 55 f.

21 Art. 1107 iran. ZGB.

22 Einzelheiten bei Bergmann/Ferid/Henrich/Enayat, a.a.O., $51 \mathrm{ff}$. und Yassari, FamRZ 2002, 1088, $1093 \mathrm{f}$.

23 Anm. 6 zu Art. 1 des Gesetzes zur Berichtigung der Scheidungsnormen (auch Scheidungsgesetz genannt), abgedruckt in Bergmann/Ferid/Henrich/Enayat, a.a.O., $139 \mathrm{f}$.

24 Ein Beispiel für nicht geschuldete Dienste ergibt sich aus Art. 1176 iran. ZGB: Eine Mutter ist nicht verpflichtet, ihr Kind zu stillen, es sei denn, die Ernährung des Kindes wäre außer durch Muttermilch nicht möglich.

25 Yassari, FamRZ 2002, 1088, 1094.

26 Bergmann/Ferid/Henrich/Enayat, a.a.O., 57.

$27 \mathrm{BGHZ}$ 160, 332 (Fn. 13).

28 BGHZ 16o, 332 Tz. 10; zustimmend: Henrich, FamRZ 2004, 1958 f.; Elwan/Menhofer, STAZ 2005, 168; Rauscher, IPRax 2005, $313 \mathrm{f.}$

29 Henrich, FamRZ 2004, 1958, 1959. 
allerdings nicht anzunehmen. Jedenfalls kann ein in Deutschland ergangenes rechtskräftiges Scheidungsurteil, das auf der Anwendung iranischen Sachrechts beruht, beim zuständigen iranischen Konsulat registriert und im Iran anerkannt werden, wenn keine der Parteien Widerspruch einlegt und das Zivile Sondergericht zwecks Überprüfung der Beachtung des iranischen Rechts anruft. ${ }^{30}$

Das Kammergericht ${ }^{31}$ hatte gleichwohl die internationale Zuständigkeit verneint und die Auffassung vertreten, ein deutsches Gericht könne die Scheidung nach dem anzuwendenden iranischen Recht nicht herbeiführen. Gegen den Willen des Ehemannes könne die Ehefrau die Scheidung nach iranischem Recht nur unter bestimmten Voraussetzungen und in einem förmlichen Verfahren dadurch erreichen, dass das Gericht den Ehemann zwinge, die Scheidungsformel auszusprechen. Gelinge dies nicht, könne nur ein „geistliches Gericht“ die Scheidung auf der Grundlage religiöser Vorschriften (Scharia) aussprechen. Da sachliches Recht und Verfahrensrecht eine untrennbare Einheit bildeten, könne ein deutsches Gericht, dem die Funktion eines geistlichen Gerichts nach Maßgabe religiöser Vorschriften wesensfremd sei, die Scheidung nicht vornehmen.

Dieser Auffassung ist der XII. Zivilsenat des Bundesgerichtshofs nicht gefolgt.

\section{Anzuwendendes Sachrecht}

Aus welchen Gründen eine Ehe geschieden werden kann, bestimmt das Scheidungsstatut. Dieses ergibt sich grundsätzlich aus der Kollisionsnorm des Art. 17 Abs. 1 EGBGB. Im Verhältnis zum Iran kommt allerdings dem Niederlassungsabkommen zwischen dem Deutschen Reich und dem Kaiserreich vom 17. Februar $1929^{32}$ Vorrang zu. Nach diesem Abkommen bleiben Parteien, die beide ein- und demselben Vertragsstaat angehören, in Familiensachen ihrem jeweiligen Heimatrecht unterworfen. Anzuwenden ist deshalb das iranische Recht. Insofern besteht aber für familienrechtliche Beziehungen kein einheitliches Rechtssystem. Da im Iran das Scheidungsrecht als Teil des religiösen Rechts angesehen wird, richten sich die Scheidungsvoraussetzungen nach den Regeln der Religionsgemeinschaft, der die Ehegatten angehören. Für die Eheleute, die schiitischen Glaubens sind, gilt deshalb das iranische ZGB in Verbindung mit dem islamischen Recht in der Ausprägung der dschafaritischen Rechtsschule der ZwölferSchia. Dieser Verweisung haben die deutschen Gerichte zu folgen. Eine Rückverweisung auf deutsches Sachrecht sieht das iranische Recht nicht vor. ${ }^{33}$

Probleme ergeben sich nun, wenn die Vorschriften des religiösen Rechts die materiellen Scheidungsvoraussetzungen mit Verfahrensvorschriften des religiösen Rechts verknüpfen, so wie es hier der Fall ist: Der Ausspruch der Scheidungsformel durch den Ehemann hat in Anwesenheit zweier gerechter Männer zu erfolgen; der religiös zuständige Richter wird eingeschaltet, wenn es bei einem Scheidungsbegehren der Ehefrau, das auf „osr wa haraj“, also eine Situation der Bedrängnis gestützt wird, nicht möglich ist, den Mann zum Ausspruch der Scheidung zu zwingen.

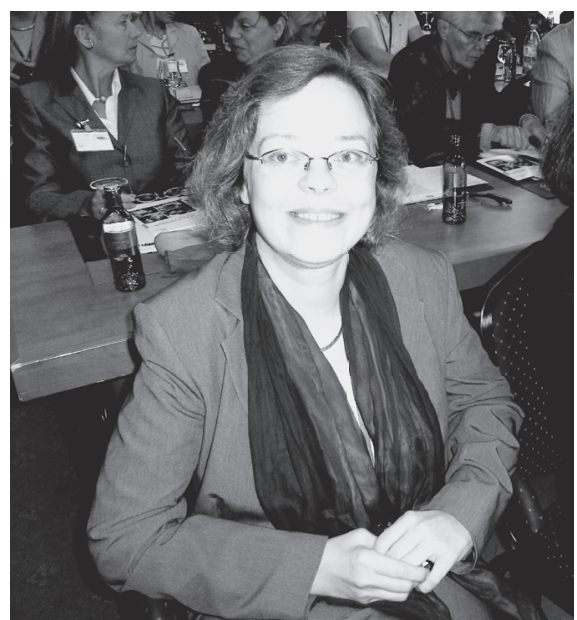

4 Richterin am BVerwG Maren Thomsen (Vorsitzende der djb-Kommission Öffentliches Recht, Europa- und Völkerrecht 2001-2005) (Foto: AG).

Insofern stellt sich die Frage, ob bei solchen sachbezogenen Verfahrensvorschriften das verfahrensrechtliche Element überwiegt. Wenn das zu bejahen ist, brauchen die ausländischen Verfahrensvorschriften an sich nicht beachtet zu werden. An ihre Stelle tritt dann das deutsche Verfahrensrecht, das die Gerichte in jedem Fall anzuwenden haben.

Der XII. Zivilsenat hat die Auffassung vertreten, dass in dem Scheidungsverfahren, für das nach iranischem Recht zunächst das zivile Sondergericht und nicht der geistliche Scharia-Richter zuständig ist, keine Aufgaben wahrzunehmen sind, die einem deutschen Gericht wesensfremd sind. Deshalb steht die Anwendung des deutschen Verfahrensrechts der internationalen Zuständigkeit auch nicht entgegen. Der SchariaRichter ist nämlich erst dann berufen, wenn es nicht möglich ist, den Ehemann zum Ausspruch der Scheidungsformel zu zwingen. Das setzt logischerweise voraus, dass er sich weigert, die Formel freiwillig auszusprechen. Allein aus der Weigerung kann die Zuständigkeit des Scharia-Richters deshalb nicht hergeleitet werden. ${ }^{34}$

Aber selbst wenn die Scheidung gegen den Willen des Mannes im Iran nur von einem religiösen Gericht durchgesetzt werden könnte, ergibt sich nichts anderes. Es ist nicht ersichtlich, welche nach iranischem Recht erforderlichen Handlungen vom anzuwendenden Recht nicht getrennt werden können und einem deutschen Gericht wesensfremd wären. Auch deutschen Gerichten ist zum Beispiel die Entgegennahme religiöser Beteuerungsformeln im Rahmen der Eidesleistung nicht fremd.

Der Umstand, dass das iranische ZGB den Ausspruch der Scheidungsformel in Gegenwart von zwei vertrauenswürdigen Zeugen männlichen Geschlechts verlangt, steht einer Verurteilung hierzu ebenfalls nicht entgegen. Auch wenn es sich nicht nur um eine Formvorschrift zum Zweck der Beweisbarkeit, sondern um ein materiell-rechtliches Wirksamkeitserfordernis handeln sollte, stellt die Form keinen Bestandteil eines religiösen Rituals dar, sondern allenfalls eine Voraussetzung der An-

\footnotetext{
30 BGHZ 160, 332 Tz. 15.

31 IPRax 2000, $126 \mathrm{ff}$.

32 RGBI. II 1930, 1002, 1006.

33 BGHZ 160, $332 \mathrm{Tz} .20 \mathrm{ff}$.

34 BGHZ 160, 332 Tz. 25 ff.
} 


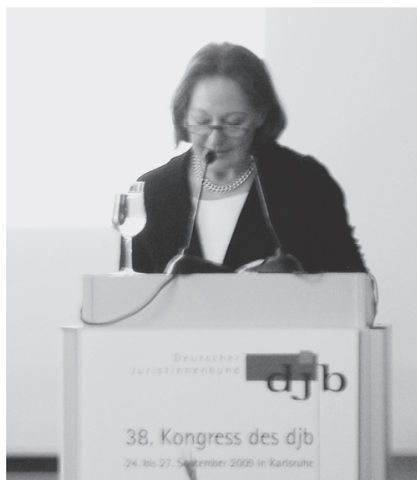

$\triangle$ Richterin am BGH Beatrix WeberMonecke (Foto: RM)

erkennungsfähigkeit der deutschen Entscheidung im Iran. Darauf Rücksicht zu nehmen ist nicht mehr, aber auch nicht weniger als ein nobile officium im Interesse des internationalprivatrechtlichen Gleichlaufs sowie zur Vermeidung hinkender Ehen.

Danach gibt es keine Hinderungsgründe, dass ein deutsches Gericht anstelle des nach iranischem Recht zuständigen Gerichts die Scheidung der Ehe ausspricht oder den Ehemann zum Ausspruch der Scheidungsformel verurteilt. Auch dernach Art. 6 EGBGB zu beachtende - deutsche ordre public steht dem nicht entgegen. Es kommt nicht darauf an, ob das iranische und das deutsche Recht auf widerstreitenden Prinzipien beruhen. Maßgebend ist vielmehr, ob das konkrete Ergebnis der Anwendung iranischen Rechts aus der Sicht des deutschen Rechts zu missbilligen ist. Das ist nicht der Fall, wenn der Ausspruch der Scheidungsformel nicht gegen den Willen der Ehefrau erfolgt, sondern weil sie einen entsprechenden Antrag gestellt hat. Im Übrigen dürfte der deutsche ordre public schon deshalb nicht verletzt sein, weil die Ehe im Hinblick auf die langjährige Trennung der Parteien auch nach deutschem Recht zu scheiden wäre. ${ }^{35}$

Wenn die internationale Zuständigkeit abgelehnt würde, käme dies auch einer Rechtsverweigerung gleich. Dies wäre im Hinblick auf den deutschen ordre public, nämlich die verfassungsrechtlich garantierte negative Eheschließungsfreiheit der Ehefrau, bedenklich, falls die Ehe auch im Iran nicht geschieden werden kann. Das kann jedenfalls nicht ausgeschlossen werden. Die Ehefrau hat geltend gemacht, bei einem persönlichen Erscheinen vor einem iranischen Gericht ein Ausreiseverbot befürchten zu müssen. Eine Scheidung ohne ihre Anwesenheit - durch Einschaltung eines Rechtsanwalts scheitert an ihrer Mittellosigkeit; ihr war in Deutschland Prozesskostenhilfe bewilligt worden. ${ }^{36}$

\section{Materiell-rechtliche Scheidungsvoraussetzungen}

Die Ehefrau hatte sowohl den Scheidungsgrund des „in Not“ oder „Bedrängnis“-Geratens geltend gemacht als auch die Weigerung des Ehemannes, Unterhalt zu zahlen. Eine Unterhaltsverweigerung setzt, wie schon erwähnt, nicht die finanzielle Leistungsfähigkeit des Ehemannes voraus. Ob dagegen mit Rücksicht auf den deutschen ordre public Bedenken bestehen, weil etwa das durch Arbeitslosigkeit bedingte Unvermögen zur Unterhaltsleistung unberücksichtigt bleibt, kann offen bleiben.
Denn die Ehe könnte auch bei Anwendung deutschen Rechts wegen des langjährigen Getrenntlebens geschieden werden. ${ }^{37}$

Der Bundesgerichtshof hat das Urteil des Kammergerichts aufgehoben und die Sache zurückverwiesen, damit erneut über das Scheidungbegehren entschieden werden konnte.

\section{Ausgang des Verfahrens nach Zurückverweisung}

Ich will Ihnen nicht vorenthalten, wie der Rechtsstreit ausgegangen ist. ${ }^{38}$ Der Ehemann ist offensichtlich ebenfalls zu der Erkenntnis gelangt, dass die Ehe gescheitert ist. Er hat die nach iranischem Recht notwendige Verstoßung durch Ausspruch der Scheidungsformel vorgenommen und hierüber die Bescheinigung einer Moschee vorgelegt. Danach konnte die Ehe durch Gestaltungsurteil geschieden werden.

Bezüglich der Kinder ist im Einvernehmen mit dem Vater folgende Regelung getroffen worden: Der Mutter ist das Personensorgerecht einschließlich des Aufenthaltsbestimmungsrechts, des Rechts, die Einbürgerung der Kinder zu beantragen, und des Rechts, Konten für sie zu eröffnen sowie die Kinder in Unterhaltsangelegenheiten zu vertreten allein übertragen worden. Im Übrigen hat der Vater die Vormundschaft behalten. Nach dem auch insofern anwendbaren iranischen ZGB hat die Mutter zwar nur bis zum 7. Lebensjahr einer Tochter sowie bis zum 2. Lebensjahr eines Sohnes den Vorrang in Bezug auf die sonst beim Vater liegende Personensorge, ${ }^{39}$ während das Kind in allen Angelegenheiten betreffend das Vermögen ohnehin der gesetzlichen Vertretung seines Vaters und des väterlichen Großvaters untersteht. ${ }^{40}$ Im Falle einer Gefährdung des Kindeswohls kann vom Gericht aber jede Entscheidung getroffen werden, „die es für die Kindessorge als notwendig erachtet". ${ }^{41}$ Mit Rücksicht darauf hat das Kammergericht angenommen, dass jedenfalls die mit dem Einverständnis des Vaters erfolgte Übertragung einzelner Rechte auf die Mutter auch nach iranischen Rechtsvorstellungen zulässig ist, ohne dass es der Feststellung einer vom Vater ausgehenden Gefährdung des Kindeswohls bedarf. Ich meine, das war eine gute Regelung. 